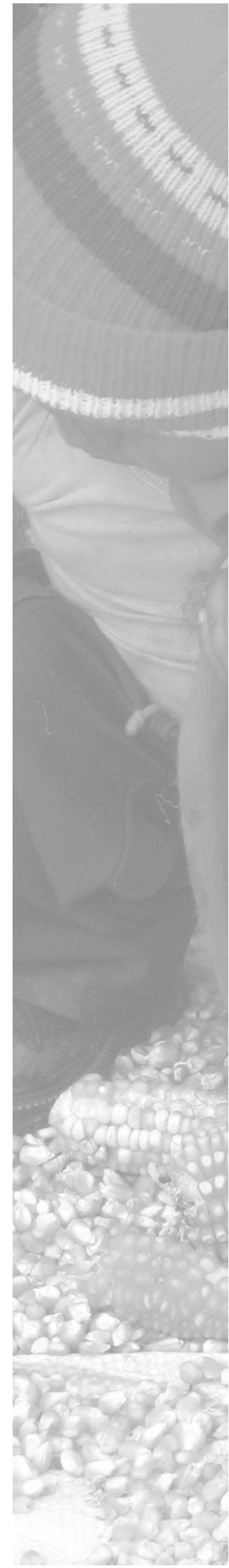

\title{
Conveniencias del
} software libre para el
Estado Colombiano

Manuel Dávila S.

\section{Resumen}

El papel de las tecnologías a nivel mundial ha afectado a las personas, las empresas, las comunidades y al mismo Estado en su vida cotidiana. De hecho, en casi todos los países y en particular en Colombia, fue creada una política nacional de Tecnologías de Información y Telecomunicaciones (TICs), con la cual se intenta regular o más bien encarrilar el desarrollo de las tecnologías de la información, para bien de la ciudadanía en general. Sin embargo, en Colombia no aparece una inclusión del software libre como tal, dejando de lado un paradigma que tiene un contexto tecnológico y social muy fuerte, debido a que por su desarrollo silencioso y no comercial, no se conocen los valores agregados que este tiene para el desarrollo de un país. Este artículo se ocupa del estudio y la argumentación con hechos, sobre el porqué el software libre debería estar presente en los planes del Estado en lo referente al proceso tecnológico, a través de un recuento de su origen, su desarrollo, los logros a nivel mundial y nacional y proponiendo mecanismos para que eso sea posible.

\section{Palabras clave:}

Software libre, Fuente abierta, Gobierno, Estado, Legislación.

\section{Abstract}

The role of technologies has affected people, enterprises, communities and the government in their daily life on a world wide scale. In fact, in almost every country, particularly in Colombia, a national policy on Information and Telecommunication Technologies (ICTs) was created. It is expected to regulate and guide the development of information technologies, for the benefit of citizens in general. However, the Colombian ICT policy does not include a role for free open source software. This ignores a paradigm that has a very strong technological and social context, because its value added towards the development of the country is not known, since free open source software is developed silently and noncommercially. This article studies and examines the facts, based on a review of the origins of free open source software, its development, and its achievements on a worldwide and national scale. The study explains why free open source software should be part of the states plans with respect to the technological process, and proposes mechanisms for achieving this goal.

\section{Key words}

Free Software, Open source, Government, State, Legislation. 


\section{Introducción}

El software se ha convertido en un elemento esencial para la vida de las personas, las empresas, las instituciones y el Estado y, es tal su importancia, que los Estados Unidos lo ha declarado como un producto de seguridad Nacional en el plan de desarrollo de la estrategia de software para el 2015 (cnsoftware. org, s.f.). En este estudio los Estados Unidos se muestran dependientes de la tecnología teniendo como eje funcional el software, toda vez que este aparece en la infraestructura crítica para el manejo de las finanzas, el transporte, las comunicaciones, las empresas y en general, en todas las actividades de los seres humanos. En esa medida, las posibles fallas del software, ponen en riesgo la infraestructura, la economía, la vida de las personas, además de generar pérdida de la confianza pública y liderazgo.

Las propuestas de dicho estudio contemplan, entre otras, mejorar la seguridad de dicha herramienta tecnológica bajo el término "software trustworthy", para educar y encaminar la fuerza de trabajo del software, estimulando la investigación y el desarrollo e innovando con representación del Gobierno, la industria y la academia.

El término "trustworthy" significa que el software debe ser digno, respetable y confiable. Así mismo, que debe ofrecer seguridad, protección, fiabilidad, rendimiento y "survivavility", asuntos que van más allá de la seguridad de las redes. El software no solo debe cumplir con su funcionalidad, sino garantizar lo anteriormente dicho.

En uno de los libros de la maestría en software libre de la Oberta de Cataluña (González, J., Pascual, J. S. \& Robles, G., 2006.), aparecen capítulos enteros dedicados a analizar la participación del Estado en este caso y de manera específica con relación al software libre.

\section{Neutralidad tecnológica en el Estado}

En forma reiterativa el Estado Colombiano, en cabeza del Ministerio de Comunicaciones, ha presentado un argumento que sería válido, si no fuera por cierto tono defensivo y es "la neutralidad tecnológica". Sin embargo, el hecho de que para el desarrollo del Plan Nacional de Tics se haya asesorado -casi de manera exclusiva- de una sola compañía, con características de monopolio o de oligopolio, equivale a hacer el Plan de Vías, con la asesoría de una empresa privada de cementos.
Teniendo en cuenta que el software libre nace de comunidades virtuales, con un altísimo contenido tecnológico y no de procesos de tipo comercial, es más compleja su inclusión, porque no depende de una "empresa", sino de las comunidades que de manera proactiva, deciden incluirlo.

Claramente, el concepto de neutralidad en tal sentido, no aplica en las decisiones actuales del Estado colombiano.

\section{El papel del software libre en la sociedad}

La penetración del software libre en las sociedades, no depende de movimientos publicitarios y mucho menos de aspectos comerciales. Su penetración solo se explica por los buenos resultados obtenidos por aquellos usuarios que han decidido usarlo y divulgarlo boca a boca, o a través de las comunidades de Internet.

Por muchos años fue solo un movimiento académico. No obstante, las universidades de los países desarrollados, siempre han influido en el mundo empresarial, lo que no sucede en Suramérica, en donde el software libre ha venido incrementando su uso en grandes compañías, que le reconocen su calidad tecnológica.

Se sabe que el software libre nace de las comunidades virtuales de Internet (es la comunidad pionera), mas no de procesos empresariales; y que, a pesar de su postura académica, ha logrado crear estándares.

Un caso muy importante es el del servidor web Apache, que cubre un $52 \%$ del mercado mundial (Netcraft, s.f.), lo que demuestra que estas comunidades tecnológicas han logrado crear "estados del arte". Además, hay más de 132.806 aplicaciones disponibles en sourceforge (sourforge.net, s.f.), uno de los repositorios de software libre más importantes.

Decidir cuáles son útiles para la sociedad es parte de la tarea que la misma sociedad debe adelantar: $y$, el Estado por su parte, tiene la responsabilidad de dar a conocer estas ventajas tecnológicas; y las universidades, las empresas privadas y oficiales deben hacer investigación aplicada, tendiente a la divulgación de tipo social.

La decisión de trabajar o no con software libre es voluntaria y este será acogido solo por quien lo desee, no hay obligaciones para hacerlo; llegar a él demanda una actitud activa y depende de la proactividad 
de los Gobiernos, comunidades, empresas, universidades y personas, a partir del conocimiento sobre su existencia.

Cuando se conoce este paradigma ocurre lo mismo que con los principios éticos: una vez identificados es una obligación promulgarlos. En general, esto sucede con todo aquello relacionado con el bien común.

\section{Algunos usuarios del software libre con trascendencia en las sociedades}

El software libre es utilizado por instituciones, empresas y personas muy influyentes en el mundo de las TICs, de los cuales se mencionan algunos para mostrar su credibilidad frente a esa alternativa.

Gigantes como Google, Wikipedia, Youtube, Flickr, Digg, entre otros, han adoptado el software libre para el manejo de sus plataformas tecnológicas, como se explica en uno de los sitios de información más conocidos de habla hispana, llamado barrapunto (softlibre.barrapunto.com, s.f.). Así mismo, existen empresas de gran tamaño como Google (s.f.), cuya plataforma utiliza la selección de Linux para 20.000 servidores que manejan 2000 búsquedas por segundo (Google, s.f.).

El software libre es la base de los servidores que ofrece IBM al mundo entero, compañía que ha liberado cerca de 500 de sus patentes de software al software libre, como un indicio de la credibilidad sobre su desempeño. Basta con buscar en Google "Software libre ibm patentes" para enterarse en más detalle de estas noticias.

La Nasa que entre otras cosas es uno de los más grandes desarrolladores de "drivers" para Linux, maneja las bases de datos de sus proveedores con Mysql, uno de los motores de bases de datos relacionales libres con muchos usuarios reportados, como se puede ver en su registro (MySQL, s.f.) que contempla datos de instituciones gubernamentales de otros países.

El líder en LMS (Learning Management Systems) o aulas virtuales de software abierto para la Educación Virtual, se llama Moodle y en Colombia lo usan cerca de 600 Instituciones (Moodle, s.f.); es muy conocido además de mencionado, en los foros del Ministerio de Educación Nacional de Colombia, pues ha impulsado la educación virtual en el país, gracias a sus capacidades tecnológicas, al uso de estándares SCORM y a que no representa costos de licenciamiento.

El gigante de los motores de bases de datos Oracle basa el desarrollo de su motor en Linux; hace unos tres años lanzó en todo el mundo, incluida Colom- bia, la iniciativa llamada "Oracle unbreakable linux" o Linux inquebrantable, otra prueba de que bajo este sistema operacional libre se manejan las bases de datos más grandes del mundo (Oracle, s.f.).

Hay instituciones colombianas inspiradas en lo social como la Corporación Universitaria Minuto de Dios (Uniminuto), que basa su plataforma tecnológica en software libre y permite a los usuarios utilizar cualquier sistema en sus computadores.

En el evento Internacional de Software Libre, realizado en Noviembre del 2007 en Bogotá, se presentó una ponencia llamada "Uniminuto, un caso de éxito en software libre", que mostró los logros obtenidos (Dávila, M., 2008). En dicha entidad educativa, se manejan 18.000 estudiantes, 19 servidores, 12 regionales remotas y más de 5.000 estudiantes conectados a sus aulas virtuales.

Igualmente, la Superintendencia de Servicios Públicos de Colombia, desde hace cinco años decidió optar por la misma alternativa y liberó el software de gestión documental Orfeo (Orfeo, s.f.) para bien de los ciudadanos, como una forma de retribuirles los impuestos, así como lo hizo Estados Unidos con el protocolo TCP/IP, base tecnológica de Internet.

La región de Extremadura de España pasó a ser una de las provincias más fuertes tecnológicamente, a pesar de ser una de las más pobres; creó una distribución de Linux llamada Linex (s.f.), como herramienta de uso libre para sus ciudadanos.

En Colombia, existen casos similares para el uso de colegios y de la ciudadanía. Algo parecido ha hecho España con el proyecto Educa Madrid (s.f.).

\section{Observaciones complementarias}

El software de oficina que contempla procesador de palabra, hoja de cálculo, y presentaciones, que en el mundo del software libre se llama Open Office, ha sido comprobadamente exitoso. Entonces, ipor qué seguir invirtiendo miles de millones de pesos en software propietario, tan costoso para las entidades del Estado, con esta realidad al alcance?

No se debe desconocer que existen casos en los que el software propietario resulta ser una solución más adecuada, pero con seguridad, se trata de una minoría a la que se le pueden suministrar las licencias requeridas.

El proceso para acceder al software libre no es el mismo que adelanta una empresa privada frente al software comercial, toda vez que se basa en comunidades libres, sin gerentes ni vendedores. 
En tal sentido, es de esperarse que si el Ministerio de Comunicaciones comprende la filosofía de este paradigma, debería buscar su inclusión, empezando por la misma entidad, buscando asesorías externas de expertos o las comunidades académicas.

De otro lado, las empresas que se constituyen en Colombia como asesoras de software libre generan empleo y el dinero de sus operaciones comerciales, basadas en el conocimiento, queda en manos de los ingenieros y tecnólogos contratados, pues se elimina el pago de licencias.

Entendiendo que el Estado necesita velocidad, como lo expresó la Ministra en una reunión con la Red de Decanos y Directores de Ingeniería de Sistemas (REDIS), vale la pena citar el concepto de Milan Kundera en su libro "La lentitud": "la velocidad es inversamente proporcional al proceso del aprendizaje" (Kundera, 1995).

¿No cabe esta frase en lo que toca con las decisiones del Estado colombiano que comprometen a millones de ciudadanos?

Otro tema que pasa inadvertido, a pesar de ser común en los medios académicos, es el relacionado con los documentos del Estado, publicados en formatos propietarios. Utilizar esta presentación es una forma de convertir al Estado en "vendedor gratuito" de marcas. Tales documentos deberían ser publicados en modelos abiertos, con el propósito de dejar en libertad a los ciudadanos para escoger las herramientas. Es necesario aclarar, que una cosa es el formato de un documento y otra, las herramientas que lo leen.

En una ocasión, preguntaron durante un foro si Bill Gates era el que se había inventado los bits. De ahí se deduce -sin demeritar a Bill Gates-, el desconocimiento del público, sobre quiénes son los creadores del estado del arte tecnológico. Ignorancia peligrosa, sobre todo, si quienes tienen en sus manos las decisiones del Estado, caen en ella.

Los Gobiernos de Brasil, Perú, Ecuador y Venezuela, entre otros países, han adoptado el software libre. El presidente brasilero, Lula Da Silva dice que invertirá lo que sea necesario para integrarlo en el Estado que él representa. Venezuela, por su parte, lo acoge con visos políticos, actitud que no es bien vista por algunos de nosotros.

Colombia no ha debatido seriamente sobre el tema, como una política de Estado y se defiende por no hacerlo, escudándose en el argumento de la "neu- tralidad tecnológica", mientras consume miles de millones de licencias de software que podría ahorrarse. No obstante, se han presentado proyectos de ley en esa dirección (slcolombia.org, s.f.).

El mismo Ballmer (2006), CEO of Microsoft, acepta a Linux como su principal competidor tecnológico, lo cual es un punto a favor del software libre. Tal es el convencimiento de Microsoft de la calidad de su competidor, que se une con Novell (s.f.) para ver cómo usa software libre en las áreas internas que muestran debilidades (Gonsalves, 2006)

En un foro sobre "Democratización de la Tecnología", en el que participamos representando a Uniminuto, al que también asistieron el ex gerente de Microsoft, Pedro Julio Uribe, el doctor Antanas Mockus, el Viceministerio de Educación, Computadores para Educar y la Agenda de Conectividad, el mismo Gerente de Microsoft se refirió a Linux como su competencia.

En la Asociación Colombiana de Ingenieros de Sistemas (ACIS), se han hecho dos Salones de Informática sobre el software libre, en jornadas de tres días, a las que han sido invitadas las multinacionales proveedoras de tecnología informática, para debatir de manera abierta este tema.

Finalmente, dar a conocer el software libre en los más de 1070 sitios en los que la Agenda de Conectividad del Estado enseña a la población sobre las tecnologías de información y las telecomunicaciones, multiplicaría el uso de la informática dentro de un contexto social, con independencia tecnológica.

Por eso se le ha propuesto al Ministerio de Comunicaciones invertir en un proyecto que nazca desde su interior, para conocer las ventajas tecnológicas con contexto social del software libre, con el fin de darle el verdadero significado a la "neutralidad tecnológica".

También se le ha insinuado al Ministerio convocar a un foro abierto sobre las conveniencias o inconveniencias del software libre para el Estado, con la participación de sus instituciones, los Ministerios de Educación y Comunicaciones, Colciencias, Agenda de Conectividad, Computadores para Educar, empresas multinacionales proveedoras de tecnología, universidades, el gremio de los Ingenieros de Sistemas, la empresa privada, las casas colombianas de software, y la Cámara de Comercio, entre otras organizaciones. Encuentro propicio para permitir que sean los argumentos y el debate, además de las conclusiones grupales y no el desconocimiento ni el "statu quo", lo que rija la toma de este tipo de decisiones. 


\section{Conclusiones}

- En este artículo se ha expuesto la conveniencia del uso del software libre por parte de la sociedad colombiana y se han dado argumentos para que sea incluido en el Plan Nacional de TICs del Ministerio de Comunicaciones de Colombia.

- Se ha explicado el origen del software libre, como parte de una comunidad tecnológica que ha logrado construir estados del arte, para lo cual se han presentado casos concretos de los resultados a nivel de las multinacionales de tecnología, los países y las organizaciones que han demostrado este paradigma como digno de tener en cuenta.

- Se ha hecho énfasis en el contexto social del software libre, fortaleza que favorece llevarlo a los lugares apartados del país, en donde la Agenda de Conectividad del Estado está llegando.

- Se ha hecho una crítica al concepto de "neutralidad tecnológica" que usa el Ministerio de Comunicaciones cuando se le propone el software libre, mientras es asesorado por una multinacional con visos de monopolio. Actitud, que parte del desconocimiento, razón por la que se le invita a un debate abierto que una al Estado, las empresas, la Academia y los ciudadanos en torno a la verdad de esta conveniencia.

\section{Referencias}

[1] Ballmer, S. (2006). Microsoft To Focus On Linux Competition, Software-As-A-Service, Internet Advertising. Recuperado el 6 de Noviembre de 2008, de http://www. informationweek.com/news/software/hosted/showArticle. jhtml?articlelD = 187202415

[2] Barrapunto (s.f.). ¿Qué plataforma usan los sitios más populares? Recuperado el 6 de Noviembre de 2008, de http:// softlibre.barrapunto.com/article.pl? Sid=07/09/07/0544231

[3] Cnsoftware.org (s.f.). A national software strategy to en- sure u.s. security and competitiveness. Recuperado el 6 de Noviembre de 2008, de http://www.cnsoftware.org/nss2report/nss2finalreport04-29-05pdf.pdf

[4] Dávila, M. (2.008). Un caso de éxito en software libre. Recuperado el 6 de Noviembre de 2008, del sitio web del Departamento de Informática, Redes y Electrónica de la Corporación universitaria Minuto de Dios:http://e-logicasoftware.com/tutoriales/conferencias/uniminuto-un-caso-deexito-en-software-libre/caso-de-exito/index.html

[5] Educa Madrid (s.f.). Recuperado el 6 de Noviembre de 2008, de http://www.educa.madrid.org/portal/web/educamadrid

[6] Gonsalves, A. (2006). Microsoft Cuts Deal With Novell To Support Suse Linux. Recuperado el 6 de Noviembre de 2008, de http://www.informationweek.com/news/software/linux/showArticle.jhtml?articlelD=193501481

[7] González, J., Pascual, J. S. \& Robles, G. (2006). Introducción al Software Libre. Recuperado el 6 de Noviembre de 2008, de http://curso-sobre.berlios.de/introsobre/sobre. $\mathrm{html} /$

[8] Google (s.f.). Google a fondo, su tecnología e infraestructura. Recuperado el 6 de Noviembre de 2008, de http://www.vnmhost.net/tips/modules. php?name=News\&fil $\mathrm{e}=$ article\&sid $=496$.

[9] Kundera, M, (1995). La lentitud. Tusquets Editores, Barcelona, España

[10] Linex (s.f.). Recuperado el 6 de Noviembre de 2008, de http://www.linex.org/joomlaex/

[1 1] Moodle (s.f). Recuperado el 6 de Noviembre de 2008, de http://moodle.org/sites/index.php?country=cO

[12] mysql (s.f.) Recuperado el 6 de Noviembre de 2008, de http://www.mysql.com/customers/

[13] Netcraft (s.f.). Falta el nombre del artículo. Recuperado el 6 de Noviembre de 2008, de http://news.netcraft.com/

[14] Oracle (s.f.). Recuperado el 6 de Noviembre de 2008, de http://www.oracle.com/technologies/linux/index.html

[15] Orfeo (s.f.). Recuperado el 7 de septiembre de 2007 , de http://orfeogpl.org/ata/

[16] Slcolombia (s.f.). Recuperado el 6 de Noviembre de 2008, de http://www.slcolombia.org/Proyecto_de_Ley?high light $=$ (ley) $\mid$ (colombia)

[17] Sourceforge (s.f.). Recuperado el 6 de Noviembre de 2008, de https://sourceforge.net/softwaremap/trove_list. php?form_cat $=18$

Manuel Dávila Sguerra. Ingeniero de Sistemas de la Universidad de Los Andes. Director del Departamento de Informática Redes y Electrónica de Uniminuto. Ex director de la Unidad de Educación Virtual, Uniminuto Virtual. Gestor y ex presidente de la Red de Decanos y Directores de Ingeniería de Sistemas (REDIS), Ex gerente de Desarrollo del Grupo Linux, S.A. Coordinador Académico de la Asociación Colombiana de Ingenieros de Sistemas (ACIS). Director del Salón de informática de ACIS, 2001 y 2004 sobre Software Libre. Columnista de Computerworld. Blog de eltiempo.com sobre Linux y el software libre. Autor de e-Genesis, El Generador de Sistemas y los textos del curso virtual sobre software libre para Bogotá, 2006.

manuel_davila@grupolinux.net 